\title{
Impacto do distúrbio do sono na qualidade de vida dos profissionais de enfermagem
}

\author{
Impact of sleep disorders on the quality of life of nursing professional \\ Impacto de los trastornos del sueño en la calidad de vida de los profesionales de enfermería
}

Tatiana de Sousa Nascimento ORCID: https://orcid.org/0000-0002-4651-1733 Centro Universitário UNINOVAFAPI, Brasil E-mail: tatyana.nascimento@hotmail.com

Taynan Mykally Marreiros de Oliveira ORCID: https://orcid.org/0000-0002-4152-5845 Centro Universitário UNINOVAFAPI, Brasil

E-mail: taynanmykallmarreiros@gmail.com

Miriam Elayne Macêdo de Sousa ORCID: https://orcid.org/0000-0002-3011-5437 Centro Universitário UNINOVAFAPI, Brasil E-mail: miryan1210@gmail.com

Bruno Rezende de Sousa ORCID: https://orcid.org/0000-0002-8734-7116 Centro Universitário UNINOVAFAPI, Brasil E-mail: brunorezendek2@gmail.com

Thaylla Joara de Brito Oliveira ORCID: https://orcid.org/0000-0001-8050-9445 Centro Universitário UNINOVAFAPI, Brasil E-mail: thayllaoliveira16@gmail.com

Amanda Lorrana Paz Costa

ORCID: https://orcid.org/0000-0002-0719-2389 Centro Universitário UNINOVAFAPI, Brasil E-mail: amandalpazz@hotmail.com

Ivonizete Pires Ribeiro

ORCID: https://orcid.org/0000-0003-0737-5430 Centro Universitário UNINOVAFAPI, Brasil E-mail: ivonizeteribeiro@gmail.com

Francisca Cecília Viana Rocha

ORCID: https://orcid.org/0000-0002-0837-6032 Centro Universitário UNINOVAFAPI, Brasil

E-mail: fceciliavr@hotmail.com

Adriana Sávia de Sousa Araújo

ORCID: https://orcid.org/0000-0003-4857-3812 Centro Universitário UNINOVAFAPI, Brasil

E-mail: adriana.araujo@uninovafapi.edu.br

\begin{abstract}
Resumo
O objetivo desse estudo foi analisar os impactos causados pelo distúrbio do sono na qualidade de vida dos profissionais de enfermagem. Para tal foi realizada uma pesquisa descritiva, quantitativa, com 40 profissionais de enfermagem que atuam nas unidades de terapia intensiva em um hospital público de Teresina-PI, nos meses de fevereiro à abril de 2021. Os questionários utilizados foram Pittsburgh Sleep Quality Index e The World Health Organization Quality Of Life-Bref, via Google Forms. Os dados coletados foram armazenados em planilhas eletrônicas do Microsoft Excel 2010 e analisados com o programa Statistical Package of Social Science versão 20.0. Como resultados tem-se que os componentes do questionário do sono mais afetados foram: qualidade subjetiva, latência, duração e disfunção durante o dia. A má qualidade do sono foi observada em $95 \%$ dos participantes, sendo a insônia apresentada em 13\%. Quanto ao questionário de qualidade de vida, $50 \%$ avaliaram-na como boa e $40 \%$ estão insatisfeitos em relação à saúde. As pontuações de cada domínio foram transformadas em uma escala de 0-100; médias mais altas sugeriram melhor qualidade de vida. O domínio mais comprometido foi o meio ambiente (relacionado ao ambiente doméstico, recursos financeiros, lazer e meio ambiente). As facetas mais afetadas foram: sono e repouso, sentimentos positivos, atividade sexual, recreação e lazer. Assim verificou-se que grande parte da amostra sofre ou já sofreu de distúrbios do sono. Desta maneira, a qualidade do sono é um fator que afeta a qualidade de vida.
\end{abstract}

Palavras-chave: Distúrbios do sono; Qualidade de vida; Profissionais de enfermagem. 


\begin{abstract}
The objective of this study was to analyze the impacts caused by sleep disorders on the quality of life of nursing professionals. To this end, a descriptive, quantitative research was carried out with 40 nursing professionals working in intensive care units in a public hospital in Teresina-PI, from February to April 2021. The questionnaires used were the Pittsburgh Sleep Quality Index and The World Health Organization Quality Of Life-Bref, via google forms. The collected data were stored in Microsoft Excel 2010 spreadsheets and analyzed with the Statistical Package of Social Science version 20.0. As a result, the components of the sleep questionnaire most affected were: subjective quality, latency, duration and dysfunction during the day. Poor sleep quality was observed in $95 \%$ of the participants, with insomnia being presented in 13\%. As for the quality of life questionnaire, 50\% rated it as good and $40 \%$ were dissatisfied with their health. The scores for each domain were transformed into a scale of 0-100; higher averages suggested better quality of life. The most compromised domain was the environment (related to the domestic environment, financial resources, leisure and the environment). The most affected facets were: sleep and rest, positive feelings, sexual activity, recreation and leisure. Thus, it was found that a large part of the sample suffers or has already suffered from sleep disorders. Thus, sleep quality is a factor that affects quality of life.
\end{abstract}

Keywords: Sleep disorders; Quality of life; Nursing professionals.

\title{
Resumen
}

El objetivo de este estudio fue analizar los impactos de los trastornos del sueño en la calidad de vida de los profesionales de enfermería. Para ello, se realizó una investigación descriptiva cuantitativa con 40 profesionales de enfermería que laboran en unidades de cuidados intensivos de un hospital público de Teresina-PI, de febrero a abril de 2021. Los cuestionarios utilizados fueron el de Índice de calidad del sueño de Pittsburgh y Quality of Life-Bref de la Organización Mundial de la Salud, a través de formularios de Google. Los datos recopilados se almacenaron en hojas de cálculo de Microsoft Excel 2010 y se analizaron con el programa Statistical Package of Social Science versión 20.0. Como resultado, los componentes del cuestionario de sueño más afectados fueron: calidad subjetiva, latencia, duración y disfunción durante el día. Se observó mala calidad del sueño en el $95 \%$ de los participantes, presentándose insomnio en el 13\%. En cuanto al cuestionario de calidad de vida, el 50\% lo calificó como bueno y el 40\% se mostró insatisfecho con su salud. Las puntuaciones de cada dominio se transformaron en una escala de 0 a 100; los promedios más altos sugirieron una mejor calidad de vida. El dominio más comprometido fue el medio ambiente (relacionado con el entorno doméstico, los recursos económicos, el ocio y el medio ambiente). Las facetas más afectadas fueron: sueño y descanso, sentimientos positivos, actividad sexual, recreación y ocio. Así, se constató que gran parte de la muestra padece o ya ha padecido trastornos del sueño. Por tanto, la calidad del sueño es un factor que incide en la calidad de vida.

Palabras clave: Trastornos del sueño; Calidad de vida; Profesionales de enfermería.

\section{Introdução}

O sono é um estado funcional, tornando-se uma das necessidades primordiais do ser humano. Um sono reparador é essencial para o equilíbrio fisiológico, após o organismo passar por um período de atividades físicas e psíquicas. Ele tem papel fundamental de relaxar e de reestabelecer o organismo para um novo ciclo de atividades, tornando-se uma atividade tranquilizadora para continuidade e qualidade de vida. Convém ressaltar que o corpo humano trabalha em formação de um relógio biológico, operando conforme as horas do dia, como, por exemplo, acordar todos os dias no mesmo horário sem despertador (Miranda \& Passos, 2020).

Cientistas apontam que há uma forte relação entre a idade e o tempo de horas de sono favorável. Dando enfoque à média da idade dos profissionais da área da saúde, os estudos realizados pela entidade Norte Americana National Sleep Foundation, afirmam que o ideal para: Adultos jovens (18 - 25 anos): entre 7 a 9 horas; - Adultos (26 - 64 anos): entre 7 a 8 horas. Dormir e não satisfazer as necessidades do corpo, principalmente, em questão de fadiga ou derivados, significa que algo está errado com o ciclo e deve ser tido como alerta (Josué Silva et al., 2019).

A qualidade de vida, diante da concepção dos autores Chiroli, Ritter e Lucio (2018), é um tema que elenca a Teoria das Necessidades de Maslow, evidenciando cinco necessidades fundamentais para o bem-estar de um indivíduo. Na base da pirâmide encontram-se as necessidades básicas do indivíduo, sendo elas fisiológicas e de segurança. O topo da pirâmide é constituído pela estima e a autorrealização. Entre as necessidades básicas e autorrealização, encontra-se o meio social, sendo fundamental para que o indivíduo domine todas as áreas. 
As consequências de um trabalho excessivo são inúmeras, interferindo diretamente, em primeira instância, na questão do desequilíbrio do sono, que implica na má qualidade de vida e no esforço mental e físico, retardando a atenção dos mesmos e aumentando os riscos de cometerem erros, por conta do cansaço, em expediente de trabalho (Josué Silva et al., 2019).

Segundo a Organização Mundial de Saúde (OMS), cerca de 40\% da população mundial não dorme como gostaria e apresenta alguns dos mais de 80 distúrbios e síndromes do sono listados na Classificação Internacional dos Distúrbios do Sono (CIDS). Além disso, os transtornos do sono representam um fator de expressivo impacto na vida de uma pessoa, causando prejuízos, em curto ou em longo prazo, às atividades diárias, às adversidades sociais, somáticas, psicológicas ou cognitivas. De acordo com uma pesquisa realizada pela Associação Brasileira do Sono, com cerca de 43 mil pessoas das principais capitais do país, mostra-se que 53,9\% delas sofrem de algum tipo de insônia (a CIDS lista 11 tipos diferentes da doença) e cerca de 43\% permanecem cansados no decorrer do dia (Aguiar et al., 2019).

O homem moderno, frente à pressão do ritmo do dia a dia, está susceptível a esses distúrbios em vários momentos da vida, porém, muitos não se atentam ao fato de estar com a qualidade do sono ruim, por seu organismo ter feito adequações para ele poder desempenhar suas funções básicas. Tendo isso como base, o objeto deste estudo são os impactos causados pelo distúrbio do sono na qualidade de vida dos profissionais de enfermagem.

Diante disso, a pesquisa tem como questão norteadora: quais os impactos do distúrbio do sono na qualidade de vida dos profissionais de enfermagem? Tal levantamento surgiu durante a observação das durações de plantões, onde, muitas vezes, os profissionais passam em média 24 horas, com descanso inadequado no ambiente de trabalho, despertando assim, curiosidade de identificar os reflexos na atuação cotidiana influenciadas pela qualidade do sono desses profissionais, e como isso pode afetar diretamente a qualidade de vida.

O objetivo geral deste estudo é avaliar os impactos causados pelo distúrbio do sono na qualidade de vida dos profissionais de enfermagem. Os objetivos específicos são: identificar os impactos causados pelo distúrbio do sono em profissionais de enfermagem; verificar os impactos gerados pelo distúrbio do sono na qualidade de vida dos profissionais de enfermagem e avaliar a associação entre o distúrbio do sono e a qualidade de vida dos profissionais de enfermagem.

Por fim, com este estudo, pretende-se conhecer e aprofundar o conhecimento sobre o assunto, que além de analisar lacunas abertas sobre o tema, também será útil para pesquisas posteriores. Poderá contribuir também para as instituições hospitalares elaborarem estratégias de intervenção que minimizem os efeitos negativos da má qualidade de sono, de modo a priorizar o bem estar dos trabalhadores. Esses profissionais poderão refletir também sobre como uma má qualidade de sono poderá afetar sua qualidade de vida.

\section{Metodologia}

Trata-se de uma pesquisa exploratória e descritiva de abordagem quantitativa, sendo realizada nas Unidades de Terapia Intensiva de um hospital público de grande porte, situado em Teresina no estado do Piauí. A população consistiu em 40 profissionais da equipe de enfermagem (amostra não probabilística de maneira intencional), sendo eles 17 Enfermeiros e 23 Técnicos de Enfermagem, alocados nos turnos manhã, tarde e noite. Como critérios de inclusão foram considerados o tempo de trabalho nas unidades de internações, igual ou maior que 12 meses. E os critérios de exclusão deste estudo foram aqueles(as) que se encontravam de férias, folga ou em licença saúde. O período de coleta de dados ocorreu em fevereiro a abril de 2021.

Após autorização da instituição coparticipante, foi solicitado junto à gerência de enfermagem o e-mail ou o telefone de contato dos participantes. Os dados foram coletados através de um formulário eletrônico, na plataforma Google Forms, enviado aos participantes do estudo. Desse modo, o formulário foi enviado através de um link, através do e-mail ou do aplicativo de mensagens WhatsApp Messenger, acessado através de computadores ou smartphones com acesso à internet. Vale 
lembrar que todos os participantes que responderam ao questionário tiveram prévia explicação sobre o objetivo deste estudo e foram esclarecidos da sua não obrigatoriedade em participar da pesquisa, podendo desistir a qualquer momento, sem serem lesionados de nenhuma forma.

Para a coleta das informações dos participantes, inicialmente aplicou-se um questionário contendo oito questões sobre seus dados sociodemográficos e ocupacionais. Em seguida foi aplicado o questionário de Pittsburgh Sleep Quality Index (PSQI) que avalia a qualidade do sono durante o último mês. O questionário possui dezenove questões autoadministrativas e cinco questões respondidas por seus companheiros de quarto. Estas últimas são utilizadas somente como informação clínica e não foram consideradas nessa pesquisa. As dezenove questões são agrupadas em sete componentes, com pesos distribuídos numa escala de 0 a 3 (Buysse et al., 1989). O PSQI possui versão validada para o português Brasil (PSQI-BR) por Bertolazi (2008).

Os componentes são: a qualidade subjetiva do sono (questão 6), a latência do sono (questões 2 e $5^{\text {a }}$ ), a duração do sono (questão 4), a eficiência habitual do sono (questões 1 e 3), distúrbios do sono (questões de 5B a 5J), uso de medicações para dormir (questão 7) e disfunção diurna (questões 8 e 9). Nesse instrumento, a soma dos escores dos sete componentes varia de 0 a 21, quanto maior o escore, pior a qualidade de sono, sendo classificado como: qualidade de sono boa ( 0 a 4$)$, qualidade de sono ruim (5 a 10) e presença de distúrbio do sono (>10) (Buysse et al., 1989).

Para avaliar a qualidade de vida foi aplicado o questionário The World Health Organization Quality Of Life-Bref (WHOQOL-BREF), sendo composto por 26 questões (as questões 1 e 2 abrangem sobre a qualidade de vida geral). Fora essas duas questões, o questionário contém 24 facetas as quais fazem parte de 4 domínios, sendo eles: físico (questões 3, 4, 10, 15, 16, 17e 18), psicológico (questões 5, 6, 7, 11, 19 e 26), relações sociais (questões 20, 21 e 22) e meio ambiente (questões 8, 9 , $12,13,14,23,24$ e 25). As respostas seguem uma escala de Likert (sendo de 1 a 5 e quanto maior for a pontuação melhor a qualidade de vida) (Whoqol Group, 1996).

Vale ressaltar que antes de fazer os cálculos as questões 3, 4 e 26 devem ser invertidas com a finalidade de padronizar todas as respostas do instrumento, de forma que, quanto mais positiva a resposta, esta deve se aproximar de 5, por conseguinte, quanto mais negativa a resposta, esta deve se aproximar de 1. A sintaxe é a média aritmética dos itens do domínio. Os escores também podem ser transformados em uma escala de 0 a 100 (adotado nesta pesquisa) onde, quanto maior o resultado melhor a qualidade de vida (Whoqol Group, 1996). A versão traduzida e validada do WHOQOL-BREF para o português do Brasil foi feita por Fleck et al. (2000).

As pontuações totais dos questionários foram analisadas e em seguida organizadas em bancos de dados eletrônicos através de digitação em planilha do aplicativo Microsoft Excel de 2010. Em seguida, foram exportados e processados para o programa Statistical Package of Social Science (SPSS) versão 20.0. A análise foi realizada através de estatística descritiva, por meio de frequências absolutas e percentuais, média, mediana, desvio-padrão e valores mínimos e máximos para que se possa descrever a realidade da população estudada. Os resultados obtidos são apresentados em forma de gráficos e tabelas.

A presente pesquisa foi submetida e aprovada pelo Comitê de Ética em Pesquisa (CEP) do Centro Universitário UNINOVAFAPI sob o parecer de número: 4.426.583, e em seguida pelo CEP do Hospital Getúlio Vargas (instituição coparticipante), sendo aprovada pelo parecer de número: 4.517.380, conforme a Resolução 466/2012 do Conselho Nacional de Saúde (CNS) (Resolução n. 466, 2012), a qual rege as diretrizes e normas regulamentadoras de pesquisas envolvendo seres humanos.

\section{Resultados}

Participaram da pesquisa 40 profissionais de enfermagem, foram analisados os dados sociodemográficos e ocupacionais apresentado na Tabela 1, nesta observou-se que o sexo que prevaleceu foi o feminino $(82,5 \%)$, o estado civil foi 
solteiro(a) (47,5\%), 55,0\% possui filho e a faixa etária foi de 30 a 43 anos (50,0\%). Em relação à formação, a maioria foram técnicos de enfermagem $(57,5 \%)$ e o tempo de formação mais observado foi de 1 a 7 anos (32,5\%). Referente aos dados ocupacionais da amostra é possível analisar que o tempo de trabalho na UTI, de 1 a 9 anos (52,5\%) foram os mais frequentes, o turno de trabalho de maior prevalência foi o da noite $(77,5 \%)$ e a maioria possui outro emprego $(60,0 \%)$.

Tabela 1. Caracterização sociodemográfica e ocupacional dos profissionais de enfermagem. Teresina-PI, 2021.

\begin{tabular}{|c|c|c|c|}
\hline & & Frequência & $\%$ \\
\hline \multirow{2}{*}{ Sexo } & Feminino & 33 & $82,5 \%$ \\
\hline & Masculino & 7 & $17,5 \%$ \\
\hline \multirow{5}{*}{ Estado Civil } & Solteiro(a) & 19 & $47,5 \%$ \\
\hline & Casado(a) & 13 & $32,5 \%$ \\
\hline & Viúvo(a) & 2 & $5,0 \%$ \\
\hline & Divorciado(a) & 2 & $5,0 \%$ \\
\hline & União estável & 4 & $10,0 \%$ \\
\hline \multirow{2}{*}{ Possui filho } & Sim & 22 & $55,0 \%$ \\
\hline & Não & 18 & $45,0 \%$ \\
\hline \multirow{6}{*}{ Faixa etária } & 23 a 29 anos & 8 & $20,0 \%$ \\
\hline & 30 a 36 anos & 10 & $25,0 \%$ \\
\hline & 37 a 43 anos & 10 & $25,0 \%$ \\
\hline & 44 a 50 anos & 4 & $10,0 \%$ \\
\hline & 51 a 57 anos & 6 & $15,0 \%$ \\
\hline & 58 a 64 anos & 2 & $5,0 \%$ \\
\hline \multirow{3}{*}{ Profissão } & Enfermeiro(a) & 17 & $42,5 \%$ \\
\hline & Técnico(a) de enfermagem & 23 & $57,5 \%$ \\
\hline & Auxiliar de enfermagem & 0 & $0,0 \%$ \\
\hline \multirow{6}{*}{ Tempo de formação } & 1 a 7 anos & 13 & $32,5 \%$ \\
\hline & 8 a 14 anos & 12 & $30,0 \%$ \\
\hline & 15 a 21 anos & 6 & $15,0 \%$ \\
\hline & 22 a 28 anos & 2 & $5,0 \%$ \\
\hline & 29 a 35 anos & 5 & $12,5 \%$ \\
\hline & 36 a 42 anos & 2 & $5,0 \%$ \\
\hline \multirow{4}{*}{ Tempo de trabalho } & 1 a 9 anos & 21 & $52,5 \%$ \\
\hline & 10 a 19 anos & 10 & $25,0 \%$ \\
\hline & 20 a 29 anos & 2 & $5,0 \%$ \\
\hline & 30 anos ou mais & 7 & $17,5 \%$ \\
\hline \multirow{3}{*}{ Turno de trabalho } & Manhã & 7 & $17,5 \%$ \\
\hline & Tarde & 2 & $5,0 \%$ \\
\hline & Noite & 31 & $77,5 \%$ \\
\hline \multirow{2}{*}{ Possui outro emprego } & Sim & 24 & $60,0 \%$ \\
\hline & Não & 16 & $40,0 \%$ \\
\hline
\end{tabular}

Fonte: Dados da pesquisa (2021).

Na Tabela 2, está representado o grupo de profissionais de enfermagem conforme as características referentes à qualidade de sono. Sobre a qualidade subjetiva, 67,5\% dos participantes consideram seu sono ruim. O componente latência do sono apresentou que $40 \%$ dos participantes demoram mais de 60 minutos para dormir; enquanto no componente duração do sono, $65 \%$ dos pacientes relataram ter de 5 a 7 horas de sono. A eficiência habitual do sono foi considerada adequada em $70 \%$ dos participantes, a frequência de distúrbio do sono foi considerada baixa em $67 \%$ da amostra e $62,5 \%$ relataram não terem feito nenhuma vez uso de medicação para dormir. A disfunção durante o dia foi relatada por 50\% dos participantes, os quais tinham dificuldades de se manterem acordados durante o dia de 3 a 4 vezes na semana, conforme é apresentado na tabela 2. 
Tabela 2. Distribuição dos profissionais de enfermagem de acordo com os componentes do questionário de PSQI-BR. Teresina-PI, 2021.

\begin{tabular}{|c|c|c|c|}
\hline & & Frequência & $\%$ \\
\hline \multirow{4}{*}{ Qualidade subjetiva do sono } & Muito boa & 1 & $2,5 \%$ \\
\hline & Boa & 7 & $17,5 \%$ \\
\hline & Ruim & 27 & $67,5 \%$ \\
\hline & Muito ruim & 5 & $12,5 \%$ \\
\hline \multirow{4}{*}{ Latência do sono } & $<$ ou $=15$ minutos & 3 & $7,5 \%$ \\
\hline & 16 a 30 minutos & 8 & $20,0 \%$ \\
\hline & 31 a 60 minutos & 13 & $32,5 \%$ \\
\hline & $>60$ minutos & 16 & $40,0 \%$ \\
\hline \multirow{4}{*}{ Duração do sono } & $>7$ horas & 2 & $5,0 \%$ \\
\hline & 6 a 7 horas & 13 & $32,5 \%$ \\
\hline & 5 a 6 horas & 13 & $32,5 \%$ \\
\hline & $<5$ horas & 12 & $30,0 \%$ \\
\hline \multirow{4}{*}{ Eficiência habitual do sono } & $>85 \%$ & 28 & $70,0 \%$ \\
\hline & 75 a $84 \%$ & 6 & $15,0 \%$ \\
\hline & 65 a $74 \%$ & 4 & $10,0 \%$ \\
\hline & $<65 \%$ & 2 & $5,0 \%$ \\
\hline \multirow{4}{*}{ Distúrbios do sono } & 0 & 0 & $0,0 \%$ \\
\hline & 1 a 9 & 27 & $67,5 \%$ \\
\hline & 10 a 18 & 11 & $27,5 \%$ \\
\hline & 19 a 27 & 2 & $5,0 \%$ \\
\hline \multirow{4}{*}{ Uso de medicação para dormir } & Nenhuma vez & 25 & $62,5 \%$ \\
\hline & Menos de $1 \mathrm{vez} / \mathrm{sem}$ & 4 & $10,0 \%$ \\
\hline & 1 a 2 vezes/semana & 4 & $10,0 \%$ \\
\hline & 3 vezes/sem ou mais & 7 & $17,5 \%$ \\
\hline \multirow{4}{*}{ Disfunção durante o dia } & Nenhuma & 1 & $2,5 \%$ \\
\hline & 1 a $2 /$ semana & 17 & $42,5 \%$ \\
\hline & 3 a 4/ semana & 20 & $50,0 \%$ \\
\hline & 5 a $7 /$ semana & 2 & $5,0 \%$ \\
\hline
\end{tabular}

Fonte: Dados da pesquisa (2021).

Procurando visualizar mais claramente os resultados, o Gráfico 1 mostra a qualidade de sono dos participantes, onde se nota o número de bons dormidores, ou aqueles que alcançaram uma pontuação de até 4 no PSQI-BR, onde apenas duas pessoas alcançaram essa pontuação. A coluna seguinte retrata os que têm padrão de sono ruim, ou seja, tiveram pontuação até 10, onde 20 pessoas foram assim classificadas. A última coluna se refere àqueles que possuem distúrbio do sono, alcançando mais de 10 pontos, em que 18 participantes atingiram essa pontuação. 
Gráfico 1. Caracterização da qualidade de sono dos profissionais de enfermagem de acordo com o PSQI-BR. Teresina-PI, 2021.

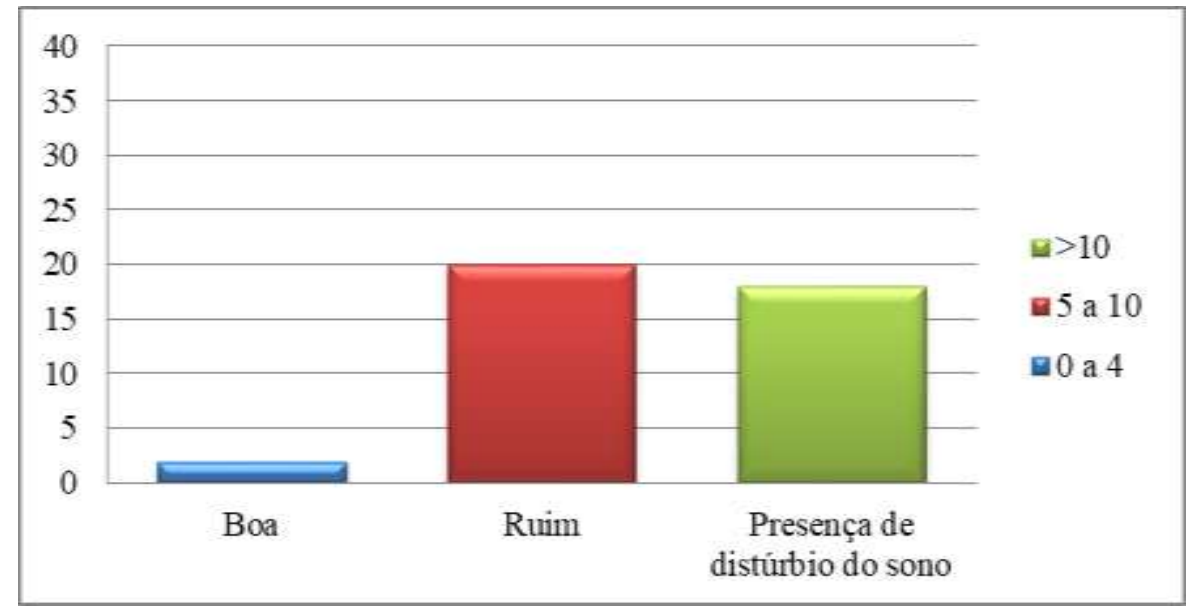

Fonte: Dados da pesquisa (2021).

É importante ressaltar alguns problemas relacionados à dificuldade de dormir, relatados por alguns participantes. Nota-se que 75\% dos participantes têm algum motivo para dificuldade de dormir, como mostra o Gráfico 2.

Gráfico 2. Motivos relatados pelos profissionais de enfermagem para dificuldade de dormir. Teresina-PI, 2021.

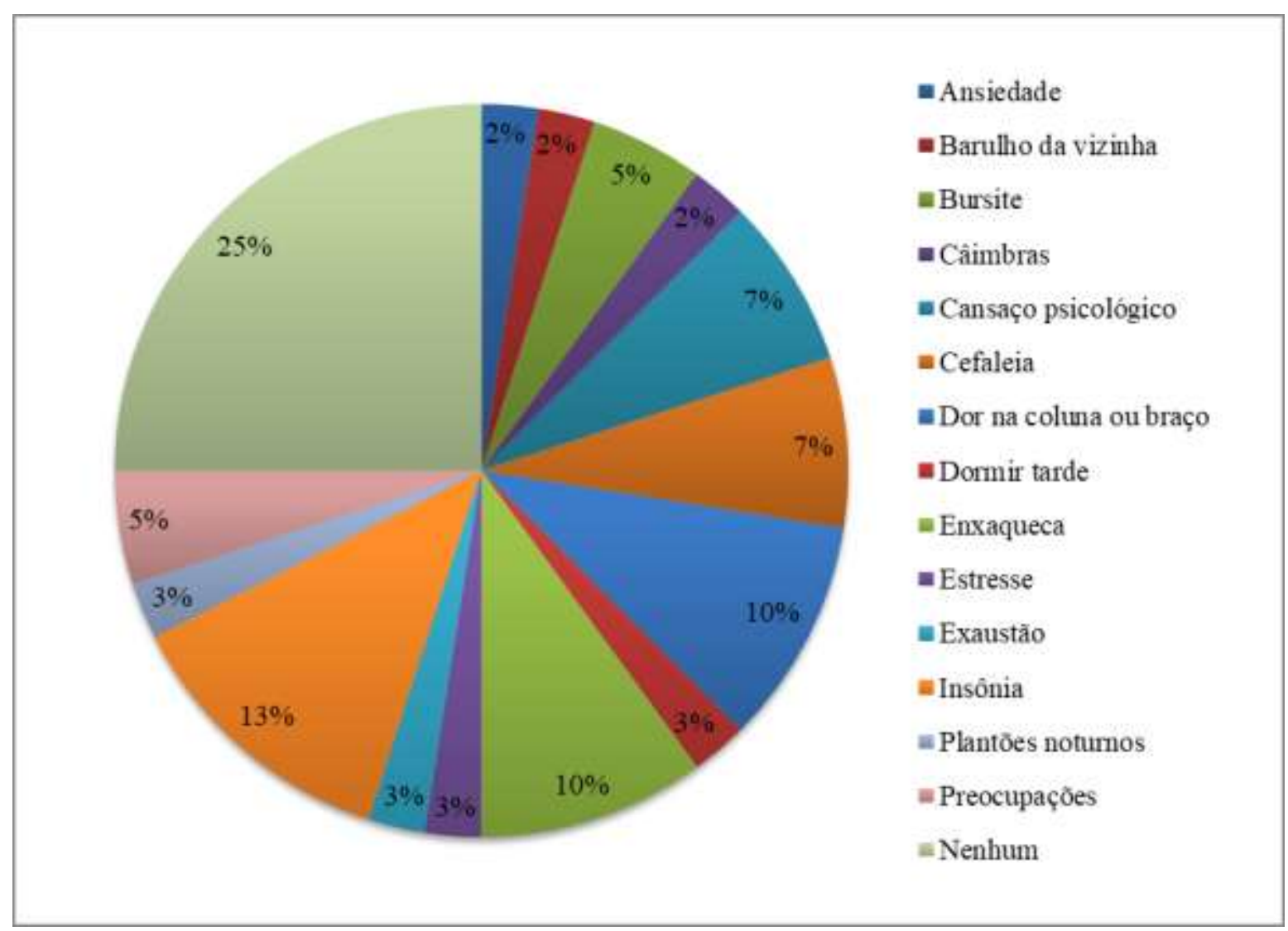

Fonte: Dados da pesquisa (2021). 
A análise descritiva referente às duas primeiras questões do questionário de WHOQOL-Bref está descrita na Tabela 3. Essas questões são gerais e não pertencem a nenhum domínio. Os resultados demonstram que 50\% avaliaram sua qualidade de vida como boa e $40 \%$ estão insatisfeitos em relação à saúde.

Tabela 3. Percepção sobre a qualidade de vida e satisfação com a própria saúde dos profissionais de enfermagem de TeresinaPI, 2021.

\begin{tabular}{|c|c|c|c|c|c|c|c|c|}
\hline Variáveis & Categorias & $\mathbf{n}$ & $\%$ & Média & Mediana & $\begin{array}{l}\text { Desvio } \\
\text { padrão }\end{array}$ & Min & Máx \\
\hline \multirow{5}{*}{$\begin{array}{l}\text { Q1: Como você } \\
\text { avaliaria sua } \\
\text { qualidade de } \\
\text { vida? }\end{array}$} & Muito ruim & 1 & 2,5 & \multirow{5}{*}{55} & \multirow{5}{*}{62,5} & \multirow{5}{*}{22,79} & \multirow{5}{*}{0} & \multirow{5}{*}{100} \\
\hline & Ruim & 9 & 22,5 & & & & & \\
\hline & $\begin{array}{l}\text { Nem ruim } \\
\text { Nem boa }\end{array}$ & 19 & 25,0 & & & & & \\
\hline & Boa & 20 & 50,0 & & & & & \\
\hline & Muito boa & 0 & 0,0 & & & & & \\
\hline Variáveis & Categorias & $\mathbf{n}$ & $\%$ & Média & Mediana & $\begin{array}{l}\text { Desvio } \\
\text { padrão }\end{array}$ & Min & Máx \\
\hline \multirow{5}{*}{$\begin{array}{c}\text { Q2: Quão } \\
\text { satisfeito(a) } \\
\text { você está com a } \\
\text { sua saúde? }\end{array}$} & Muito Insatisfeito & 1 & 2,5 & \multirow{5}{*}{47,5} & \multirow{5}{*}{50} & \multirow{5}{*}{23,20} & \multirow{5}{*}{0} & \multirow{5}{*}{100} \\
\hline & Insatisfeito & 16 & 40,0 & & & & & \\
\hline & $\begin{array}{l}\text { Nem satisfeito Nem } \\
\text { insatisfeito }\end{array}$ & 9 & 22,5 & & & & & \\
\hline & Satisfeito & 14 & 35,0 & & & & & \\
\hline & Muito satisfeito & 0 & 0,0 & & & & & \\
\hline Total & & 40 & 100 & & & & & \\
\hline
\end{tabular}

Fonte: Dados da pesquisa (2021).

A Tabela 4 mostra que o domínio mais afetado, apresentando menor escore médio, foi o meio ambiente $(56,1)$, seguido por psicológico $(58,2)$ e físico $(60,8)$. O domínio das relações sociais $(66,3)$ foi o que apresentou maior escore médio.

Tabela 4. Distribuição dos profissionais de enfermagem de acordo com os domínios do questionário de WHOQOL-Bref. Teresina-PI, 2021.

\begin{tabular}{cccccc}
\hline Domínios & Média & Mediana & Desvio Padrão & Min & Máx \\
\hline Físico & 60,8 & 60,7 & 11,9 & 35,7 & 82,1 \\
Psicológico & 58,2 & 62,5 & 14,9 & 25 & 79,1 \\
Relações Sociais & 66,3 & 66,6 & 14,6 & 33,3 & 91,7 \\
Meio Ambiente & 56,1 & 56,3 & 10,8 & 18,8 & 78,1 \\
\hline
\end{tabular}

Fonte: Dados da pesquisa (2021).

Em relação às facetas dos quatro domínios do WHOQOL-Bref, conforme a Tabela 5 observa-se que, no domínio físico, o sono e o repouso foram os que alcançaram menor média (44,38). No domínio psicológico, sentimentos positivos $(46,25)$ foi o que alcançou menor média. No que tange ao domínio das relações sociais, a menor média ficou em atividade sexual (54,38); enquanto no domínio meio ambiente, as menores médias foram recreação/lazer $(35,00)$. 
Tabela 5. Distribuição dos profissionais de enfermagem por facetas dos domínios do WHOQOL-Bref. Teresina-PI, 2021.

\begin{tabular}{lcc}
\hline \multicolumn{1}{c}{ Questões WHOQOL-Bref } & Média & Desvio Padrão \\
\hline Domínio 1 - Físico & & 21,93 \\
Q3 - Dor e desconforto & 62,50 & 25,29 \\
Q4 - Dependência de medicação ou tratamento & 63,75 & 16,98 \\
Q10 - Energia e fadiga & 50,00 & 25,29 \\
Q15 - Mobilidade & 73,75 & 24,99 \\
Q16 - Sono e repouso & 44,38 & 23,20 \\
Q17 - Atividade da vida cotidiana & 60,00 & 18,39 \\
Q18 - Capacidade para o trabalho & 71,25 & \\
\hline Domínio 2 - Psicológico & & 20,84 \\
Q5 - Sentimentos positivos & 46,25 & 22,32 \\
Q6 - Espiritualidade & 78,75 & 22,65 \\
Q7 - Pensar, memória, concentração, aprender & 50,00 & 20,84 \\
Q11 - Imagem corporal & 58,75 & 26,18 \\
Q19 - Autoestima & 58,13 & 30,06 \\
Q26 - Sentimentos negativos & 57,50 & \\
\hline Domínio 3 - Relações sociais & & 18,56 \\
Q20 - Relacionamentos pessoais & 68,75 & 29,35 \\
Q21 - Atividade sexual & 54,38 & 27,67 \\
Q22 - Suporte (apoio) social & 60,63 & \\
\hline Domínio 4 - Meio Ambiente & & 20,25 \\
Q8 - Segurança física e proteção & 52,50 & $25,03,01$ \\
Q9 - Ambiente físico, poluição ruído, trânsito, clima & 37,55 & 20,36 \\
Q12 - Recursos financeiros & 45,00 & 19,08 \\
Q13 - Oportunidades de adquirir novas informações & 58,13 & \\
Q14 - Recreação/Lazer & 35,00 & 75,63 \\
Q23 - Moradia & 65,63 & 78,75 \\
Q24 - Cuidados de saúde e sociais & & \\
Q25 - Transporte & & \\
\hline
\end{tabular}

Fonte: Dados da pesquisa (2021).

\section{Discussão}

Por meio dessa investigação e analisando os dados sociodemográficos apresentados na tabela 1, notou-se que o sexo predominante foi o feminino (82,5\%). Isso pode ser justificado pelo contexto histórico da enfermagem, pois, há muitas décadas, o setor saúde é predominantemente feminino. A Enfermagem, tradicionalmente, sempre contribuiu para essa feminilização da saúde. Os dados da pesquisa realizada pelo Conselho Federal de Enfermagem (COFEN, 2015), confirmam essa assertiva, em que $84,6 \%$ eram mulheres.

Em relação ao estado civil, a pesquisa mostra que 42,5\% dos sujeitos eram solteiros e 55\% possuíam filhos, corroborando a pesquisa de Josué Silva et al. (2019), que encontraram resultados de solteiros (45,5\%), com filhos (65,5\%), o que demanda muito no âmbito doméstico também. Costa et al. (2017) e V. S. Souza et al. (2018) pontuam as tarefas domésticas como um fator que também pode interferir na qualidade vida, uma vez que demanda da pessoa responsabilidades e deveres no âmbito particular.

É possível observar também que a faixa etária de maior predominância foi de 30 a 43 anos (50,0\%), a este respeito na pesquisa de Carvalho, Cardoso, Silva, Lira e Carvalho (2018), os trabalhadores estavam na faixa etária de 33 a 45 anos e, no estudo de Josué Silva et al. (2019), os profissionais tinham entre 31 e 40 anos. De modo geral, esses resultados expressam a disponibilidade de pessoas relativamente adulto-jovens ingressando no mercado de trabalho em saúde.

A maioria dos entrevistados foram técnicos de enfermagem (57,5\%), o predomínio de técnicos de enfermagem se justifica pelo fato de constituir o maior número de profissionais da equipe, o que corrobora os dados do COFEN (2021), onde 
57,9\% são técnicos de enfermagem. Segundo Carvalho et al. (2018), o serviço do estudo oferece incentivo à formação continuada e isso possibilitou que a maioria dos auxiliares de enfermagem fizessem uma complementação para técnicos.

O tempo de formação mais observado foi de 1 a 7 anos (32,5\%). Outros estudos com profissionais que trabalham em UTI, também relataram esses dados, nos quais se nota que o tempo de permanência destes profissionais nesta atividade é reduzido. Estes resultados sugerem que os profissionais mais experientes mudam de atividade ao longo dos anos, provavelmente, para atividades com menor nível de estresse e desgaste emocional (Guerra et al., 2016).

Ainda sobre a tabela 1, referente aos dados ocupacionais, percebeu-se que 52,5\% têm o tempo de trabalho entre 1 e 9 anos na UTI, um ambiente com alta demanda e exigência; a rotina de trabalho nesses setores é marcada pela alta complexidade e expõe a equipe de enfermagem ao estresse prolongado e acarreta repercussões à segurança do paciente e a qualidade do cuidado prestado (Souza et al., 2018). O tempo de atuação profissional pode ser um fator de proteção para a qualidade de vida no trabalho, quando a organização oferece subsídios, como melhores salários, salubridade e respeito, pois o profissional se sente útil e valorizado. Todavia, nos cenários em que o serviço não se preocupa com a saúde do trabalhador, o aumento do tempo de serviço só gera desgaste, estresse e adoecimento (Carvalho et al., 2018).

Percebeu-se, também, que a maioria dos participantes da pesquisa trabalhavam no turno da noite $(77,5 \%)$, o que pode interferir nos padrões e nos ciclos do sono. A privação do sono, segundo Soares et al. (2018), diminui a responsividade do cérebro, refletindo na diminuição do desenvolvimento cognitivo e psicomotor. Um estudo apontou que trabalhadores do turno diurno obtiveram melhor desempenho em testes de atenção, após 12 horas de trabalho, quando comparados com os resultados dos participantes do noturno.

Ainda, 60\% dos participantes possuíam outro emprego, o que, por sua vez, pode aumentar significativamente a sobrecarga de trabalho. De acordo com Costa et al. (2017) e Souza et al. (2018), a sobrecarga de trabalho é um fator que afeta profundamente a qualidade de vida dos profissionais. No âmbito da enfermagem, a sobrecarga de trabalho é uma realidade concreta por estarem sob alta exigência e demanda. Jonathan Silva et al. (2020) também verificaram que 73\% dos participantes possuem mais de um emprego na área da saúde. Esse dado pode estar ligado à necessidade financeira dos profissionais diante da baixa remuneração e da desvalorização da profissão.

Todos esses dados, tanto os sociodemográficos como os ocupacionais, são variáveis importantes no que diz respeito às investigações acerca da qualidade do sono, pois podem manter diferentes relações com a forma como o sono se desenrola em seus padrões e processos. A relação dessas variáveis com a qualidade do sono e de vida dos profissionais de enfermagem pode ser verificada conforme as estatísticas contidas nas tabelas mencionadas.

Acerca dos componentes que envolvem o questionário do PSQI-BR (Tabela 2), utilizado para avaliar as condições de sono dos indivíduos durante o último mês, verificou-se que 67,5\% dos participantes consideram seu sono ruim (qualidade subjetiva do sono). A eficiência habitual do sono foi considerada adequada em $70 \%$ dos participantes; a frequência de distúrbio do sono foi considerada baixa em $67 \%$ da amostra e $62,5 \%$ relataram que não fizeram nenhuma vez uso de medicação para dormir.

O componente latência do sono mostrou que $40 \%$ dos participantes demoram mais de 60 minutos para dormir e que 65\% possuem duração do sono entre 5 a 7 horas, sendo que, segundo Josué Silva et al. (2019) a latência do sono em um indivíduo normal seria de 10 a 30 minutos e o recomendável seria dormir em média de 7 a 8 horas, sua alteração pode resultar em disfunção durante o dia, a qual foi encontrada em $50 \%$ dos profissionais.

Em seu estudo com 66 profissionais de enfermagem Josué Silva et al. (2019) encontram que 74,5\% não utilizam remédios para dormir. Todavia, observou-se que a sonolência diurna, a duração do sono e a latência do sono, foram os fatores que mais contribuíram para a queda na qualidade do sono dos profissionais de enfermagem. Portanto, o tempo de descanso, 
latência do sono, bem como a sonolência diurna, podem afetar o tempo de sono e também sua qualidade geral, confirmando o potencial estressante do contexto de trabalho e sua interface com a saúde dos profissionais de enfermagem.

O gráfico 1 mostra o escore do questionário do PSQI-BR, numa escala de 0 (melhor qualidade) a 21 (pior qualidade), em que a qualidade de sono ruim (45\%) e a presença de distúrbios do sono (50\%) foram predominantes na maioria dos participantes. Jonathan Silva et al. (2020), encontraram em seu estudo que a maioria dos profissionais de enfermagem têm o sono ruim (52\%) e outros apresentam distúrbios do sono (36\%). Guerra et al. (2016) também observaram má qualidade de sono desses profissionais e Josué Silva et al. (2019) relataram que 56\% desses profissionais apresentaram baixa qualidade do sono, sendo assim, associada a erros simples na assistência prestada durante o turno de trabalho. Assim, é notório que os profissionais de enfermagem possuem qualidade de sono ruim e que isso pode acarretar problemas na qualidade de vida desses profissionais, pois o mesmo se alterado, pode afetar o equilíbrio de todo o organismo a curto, a médio e, mesmo, em longo prazo.

Não há dúvida de que uma boa qualidade de sono tem efeito positivo sobre a saúde e o bem-estar do ser humano. Muitas vezes, o tempo limitado e os recursos inadequados em ambientes hospitalares, podem criar sérios problemas para a promoção da qualidade do sono e tratamento dos distúrbios do sono. O impacto destes é fundamental para avaliar a qualidade de vida de uma população (Viana et al., 2019).

No que diz respeito aos motivos de dificuldade para dormir (gráfico 2), é notável que houve predominância dos seguintes motivos: insônia (13\%), seguido por enxaqueca (10\%), dor na coluna ou braço (10\%), cefaleia (7\%) e cansaço psicológico (7\%), como mostra o gráfico 2. A insônia foi o motivo mais citado pelos participantes. Conforme a International Classification of Sleep Disorders 3 (ICSD, 2014), a insônia faz parte das sete categorias principais dos distúrbios do sono. Caracteriza-se como uma dificuldade de iniciar ou manter o sono, causando cansaço durante o dia, podendo provocar prejuízos nas dimensões pessoais, sociais e profissionais, como o aumento da irritabilidade diurna, má concentração e redução da atenção, que podem estar relacionadas a taxas de acidentes mais elevadas.

De acordo com Moraes, Martino e Sonati (2018), os profissionais de enfermagem necessitam ter uma boa qualidade de vida. Pois, para que pudessem exercer suas atividades de forma humanizada. É importante manter a boa qualidade de vida. Além disso, os mesmos destacam que nesse processo estão envolvidos aspectos subjetivos (dimensões pessoais e perceptivas) e aspectos objetivos (dimensões ambientais e ocupacionais) na qualidade de vida do enfermeiro. Conforme exposto acima e com vários estudos, nota-se a insatisfação dos profissionais da enfermagem com seu sono, causando impacto na qualidade de vida. Diante dessa realidade de impacto do sono na qualidade de vida, neste estudo, será feita a análise geral dos escores do WHOQOL-Bref.

Assim, sobre essa questão, de modo geral, a tabela 3 apresenta as questões abertas referentes à qualidade de vida dos sujeitos pesquisados: 50\% avaliaram sua vida como boa e 40\%, no entanto, estão insatisfeitos com a saúde. Em contrapartida, Oliveira, Silva, Moraes e Guido (2016) encontrou em sua pesquisa com 50 profissionais de enfermagem que os mesmos avaliam sua qualidade de vida como nem ruim, nem boa e que não se sentem, nem insatisfeitos, nem satisfeitos com a sua saúde. Os resultados da pesquisa de R. S. Souza et al. (2020) evidenciaram que a maioria dos participantes avaliaram sua qualidade de vida global como "boa" ou "muito boa" $(80,4 \%)$, prevalecendo os que afirmaram estar "satisfeitos" com a própria saúde $(54,0 \%)$.

Em um estudo realizado por Trzimajewski et al. (2020) foi visto que 22 dos 32 profissionais de enfermagem entrevistados avaliam sua qualidade de vida como boa, seguindo de sete profissionais que avaliam sua qualidade de vida como, nem ruim, nem boa e a respeito da satisfação pessoal com a saúde foi evidenciado que, dos 32 profissionais participantes, 15 estão satisfeitos com sua saúde, seguindo de 13 profissionais que relatam não estarem nem satisfeitos, nem insatisfeitos.Com isso é possível notar que, a literatura encontrada reforça os resultados apresentados nesta pesquisa, em relação à qualidade de 
vida geral dos profissionais de enfermagem, os quais consideram mais "boa" do que "ruim", e que, em relação a sua saúde, eles estão, em média, satisfeitos.

De modo geral, ao analisar os resultados dos domínios do questionário de WHOQOL-Bref (Tabela 4), vale ressaltar que o mesmo é avaliado numa escala de 0 a 100, onde quanto maior o resultado melhor a qualidade de vida. É possível observar que o domínio mais afetado, apresentando menor escore médio foi o meio ambiente $(56,1)$, seguido por psicológico $(58,2)$, e os que apresentaram melhor qualidade de vida foram: relações sociais $(66,3)$ e físico $(60,8)$.

Corroborando com esse estudo, um estudo realizado por Oliveira et al. (2016) com 55 profissionais de equipe de enfermagem de um hospital municipal do Noroeste MT e outro realizado por Queiróz, Ferreira e Azevedo (2019) em seu estudo com 35 funcionários do serviço de enfermagem, de um hospital Geral em São Paulo, apresentaram menor média de qualidade de vida no domínio meio ambiente e maior média em relações sociais. Em uma pesquisa realizada por Araújo et al. (2018) com 19 profissionais de enfermagem, encontraram os domínios físicos e meio ambiente com menor escores médios. Moraes et al. (2018) também em seu estudo com 226 profissionais de enfermagem, que trabalhavam em UTI adulto de seis hospitais gerais, encontraram menor média no domínio meio ambiente.

O domínio relações sociais corresponde aos relacionamentos sociais, atividade sexual e apoio social, assim a pesquisa mostra que os profissionais de enfermagem estão mais satisfeitos com esses aspectos do que com o domínio meio ambiente que aborda segurança física e proteção, ambiente físico (poluição, ruído, trânsito, clima), recursos financeiros, oportunidades de adquirir novas informações, recreação/lazer, moradia, cuidados de saúde e sociais e transporte. Assim, um ambiente em uma UTI pode ser estressante para os profissionais, devido à alta tecnologia dos aparelhos com os quais lidam, as mudanças repentinas no estado geral do paciente e as emergências frequentes, além do contato com a mortes, esses profissionais podem apresentar esgotamentos físicos e psíquicos, afetando diretamente o sono e a sua qualidade de vida e, consequentemente, a assistência prestada.

Referente aos resultados conforme as facetas de WHOQOL (Tabela 5), é possível notar que no domínio físico, a faceta sono e repouso foi o que alcançou menor média $(44,38)$. Na pesquisa realizada por Moraes et al. (2018), seus dados evidenciaram que o sono e o repouso tinham causado insatisfação na maioria dos participantes da amostra e destacam também, a privação do sono como um fator predominante entre os profissionais da enfermagem.

Nessa mesma linha, Mello, Machado, Amorim, Almeida e Almeida (2019), ao investigar a qualidade de vida de enfermeiros, destacada a insatisfação em relação ao sono no domínio físico. $\mathrm{O}$ autor ainda diz que o sono é um fator de grande importância, pois quando o mesmo está alterado, há a possibilidade de alterações no ritmo circadiano, o que gera impacto na qualidade de vida, diminuindo o estado de alerta, o desempenho e a capacidade de aprendizado, com maior risco de ferimentos e acidentes de trabalho e diminuição na qualidade da assistência aos pacientes. Araújo et al. (2018) também encontraram, em sua pesquisa, que a faceta sono e repouso apresentou escore médio baixo, o que é preocupante, pois padrões anormais de sono causam irritabilidade, impaciência, indisponibilidade e desânimo, com impacto direto na qualidade de vida e associação com transtornos como estresse, ansiedade e depressão.

No que diz respeito ao domínio Psicológico, a faceta sentimentos positivos apresentou menor média $(46,25)$, ou seja, esses participantes consideram que não estão aproveitando a vida o suficiente. A jornada dupla de trabalho, presente em $60 \%$ dos entrevistados, pode estar relacionada e contribuir para essa insatisfação, pois os trabalhadores de enfermagem são submetidos a cargas de trabalho intensas e estressantes, além de atuarem em turnos de longa jornada e em fins de semana e feriados, privando-se de outras atividades, como as de lazer e aquelas em família, com perda na qualidade de vida, do sono e do humor. Diferentemente de Queiróz et al. (2019) e Souza et al. (2020) onde se encontraram escore médio na faceta de sentimentos negativos. $\mathrm{O}$ ambiente de trabalho pode interferir nesse resultado. 
O domínio das relações sociais verificou baixa média apenas na faceta atividade sexual. Moraes et al. (2018) afirmam que a profissão de enfermagem tem quadros de horários incompatíveis com os quadros de horários de outras pessoas de sua família, cônjuges e amigos, impossibilitando um bom desenvolvimento das relações sociais. Diferente de Queiróz et al. (2019) e Souza et al. (2018), que destacaram dados que evidenciam um alto nível de satisfação com todas as facetas desse domínio.

Por fim, acerca do domínio Meio Ambiente, a faceta recreação/lazer, foi a que apresentou menor escore médio (35,00). Nessa mesma perspectiva, os dados achados por Moraes et al. (2018), apontam pouca relevância dada pelos participantes à faceta recreação/lazer. Araújo et al. (2018) também obtiveram em sua pesquisa escores com média baixa na faceta recreação e lazer. O trabalho em turno de 12 horas interfere tanto na qualidade do sono quanto nas atividades de vida diária dos profissionais de enfermagem, principalmente as lotadas no turno noturno. Frequentemente, perdem eventos especiais com amigos e familiares, o que prejudica suas atividades de lazer e o fortalecimento das relações de apoio social.

Em síntese, foi possível observar, mesmo tendo em vista as limitações do presente estudo, pois se trata de uma amostra específica em condições contextuais específicas, a relevância dos dados achados e discutidos. Foi possível evidenciar variáveis semelhantes a de outros estudos, assim como variáveis particulares da amostra pesquisada. Diante disso, atesta-se a validade da presente investigação, uma vez que foram demonstrados aspectos que interferem na qualidade de vida dos profissionais de enfermagem.

\section{Conclusão}

O sono é muito importante para o equilíbrio do corpo e da mente humana, pois o mesmo é o principal mecanismo de homeostase do organismo, tendo papel importante na função cerebral. Durante este período, as condições orgânicas são reparadas, junto a outas funções desenvolvidas, como a reorganização dos aprendizados adquiridos durante o dia. Com isso, um bom sono e um sono adequado são essenciais para a saúde e o bem-estar do indivíduo e sua qualidade de vida.

O estudo apresenta resultados significativos acerca do sono e qualidade de vida, em que foram mostrados fatores que podem caracterizar alterações na qualidade de vida do indivíduo, comprometendo assim o contexto profissional, social, levando ao desfecho da saúde.

Portanto, a pesquisa bibliográfica, junto à compreensão e a percepção dos entrevistados desta pesquisa, foi possível verificar evidências de que grande parte da população sofre ou já sofreu de distúrbios do sono, sendo o mais comum a insônia. Desta maneira, a qualidade do sono é um fator que afeta diretamente a qualidade de vida.

Este estudo apresentou algumas limitações, dentre elas pode-se citar o contexto em que a sociedade vive atualmente, que é o de pandemia do COVID-19, o qual interferiu na coleta de dados, onde muitos trabalhadores de enfermagem foram afastados de seus devidos cargos, nos impossibilitando de chegar a um maior número de participantes.

Acredita-se que este estudo possa despertar e incentivar o interesse das pessoas acerca do tema, encorajando novas pesquisas para explorar o tópico da qualidade do sono e as relações existentes com a qualidade de vida dos profissionais de enfermagem, desencadeando a reflexão e atitudes que influenciem, de forma positiva, a saúde desses profissionais, além de priorizar medidas promocionais e preventivas de qualidade de vida dentre a importância desse tema. Pois, a má qualidade do sono, a insônia e os demais distúrbios. Faz-se necessário diagnosticar, tratar ou orientar, para não prejudicar a vida cotidiana na sociedade.

\section{Referências}

Aguiar, G. P. S., Dussán-Sarria, J. A., \& Souza, A. de (2019). Alterações do sono em pacientes vivendo com o vírus da imunodeficiência humana e dor crônica. $\operatorname{BrJP}, 2(2), 123-131$. 
American Academy of Sleep Medicine. (2014). International Classification of Sleep Disorders: Diagnostic and Coding Manual (3aed). Westchester. http://www.aasmnet.org/store/product.aspx?pid=849.

Araújo, F. D. P., Brito, O. D., Lima, M. M. de S., Galindo, N. M., N., Caetano, J. Á., \& Barros, L. M. (2018). Avaliação da qualidade de vida dos profissionais de enfermagem do atendimento pré-hospitalar. Revista Brasileira de Medicina do Trabalho, 16(3), 312-317.

Bertolazi, A. N. (2008). Tradução, adaptação cultural e validação de dois instrumentos de avaliação do sono: Escala de Sonolência de Epworth e Índice de Qualidade de Sono de Pittsburgh.

Buysse, D. J., Reynolds III, C. F., Monk, T. H., Berman, S. R., \& Kupfer, D. J. (1989). The Pittsburgh Sleep Quality Index: a new instrument for psychiatric practice and research. Psychiatry research, 28(2), 193-213.

Carvalho, A. de M. B., Cardoso, J. A., Silva, F. A. A. da, Lira, J. A. C., \& Carvalho, S. M. (2018). Qualidade de vida no trabalho da equipe de enfermagem do centro cirúrgico. Enfermagem em foco, 9(3).

Chiroli, D. M. de G., Ritter, G. J., \& Lucio, T. B. (2018). Identificação da qualidade de vida e qualidade de vida no trabalho de profissionais da área de saúde. Revista Uningá, 55(1), 177-194.

Conselho Federal de Enfermagem - COFEN. (2021). Enfermagem em números. http://www.cofen.gov.br/enfermagem-em-numeros.

Conselho Federal de Enfermagem - COFEN. (2015). Pesquisa do perfil da enfermagem brasileira. http://www.cofen.gov.br/pesquisa-inedita-traca-perfildaenfermagem_31258.html.

Costa, K. N. de F. M., Costa, T. F. da, Marques, D. R. F., Viana, L. R. de C., Salviano, G. R., \& Oliveira, M. S. de. (2017). Qualidade de vida relacionada à saúde dos profissionais de enfermagem. Rev. enferm. UFPE on line, 881-889.

Fleck, M., Louzada, S., Xavier, M., Chachamovich, E., Vieira, G., Santos, L., \& Pinzon, V. (2000). Aplicação da versão em português do instrumento abreviado de avaliação da qualidade de vida" WHOQOL-bref". Revista de saúde pública, 34, 178-183.

Guerra, P. C., Oliveira, N. F., Terreri, M. T. de S., Ascensão, L. R., \& Len, C. A. (2016). Sono, qualidade de vida e humor em profissionais de enfermagem em Unidades de Terapia Intensiva Infantil. Revista da Escola de Enfermagem da USP, 50, 0279-0285.

Mello, D. G. de, Machado, T. de S., Amorim, T. V., Almeida, É. B. de, \& Almeida, D. B. L. de (2019). Qualidade de vida no trabalho da equipe de Enfermagem intensivista em um hospital de ensino. Revista de Enfermagem da UFJF, 5(1), 1-14.

Miranda, I. P. V., \& Passos, M. A. N. (2020). Sono: fator de risco para a qualidade de vida do profissíonal de saúde. Revista JRG de Estudos Acadêmicos, 3(7), 336-346.

Moraes, B. F. M., Martino, M. M. F. de, \& Sonati, J. G. (2018). Percepção da qualidade de vida de profissionais de enfermagem de terapia intensiva. Revista Mineira de Enfermagem, 22, 1-6.

Oliveira, G. M. de, Silva, R. M. da, Moraes, I. M. de, F., \& Guido, L. de A. (2016). Influência do turno de trabalho na qualidade de vida dos profissionais de enfermagem de um hospital público do noroeste do Mato Grosso-MT. Revista de Divulgação Científica Sena Aires, 5(1), 4-20.

Queiróz, B. R. S. de, Ferreira, M. da G., \& Azevedo, O. A. de (2019). Qualidade de vida do profissional de enfermagem que atua em uma instituição hospitalar de rede pública. Life Style, 6(1), 31-46.

Resolução Conselho Nacional de Saúde n. 466, de dezembro de 2012. Trata de pesquisas em seres humanos e atualiza a resolução 196. Diário Oficial da União. Brasília, DF. http://conselho.saude.gov.br/resolucoes/2012/Reso466.pdf.

Silva, J. R. de C. S. [Jonathan], Ykeda, D. S., Medeiros, D. C. N. de, \& Costa, R. A. de O. (2020). Avaliação da qualidade do sono e nível de sonolência de profissionais da enfermagem. In S. R. M. Barbosa. Tecnologia e inovação para o cuidar em enfermagem. (pp. 68-78). Atena.

Silva, J. da S. X., Silva, R. M., Cangussu, D. D. D., Moraes, I. M. de, F., Pérez, M. A., \& Proença, M. F. R. (2019). Qualidade do sono dos profissionais de enfermagem do serviço móvel de atendimento de urgência. Revista de Divulgação Científica Sena Aires, 8(3), 264-272.

Soares, C. G., Mello, M. C. V. A. de, Santos, K. N. S. C., Modernel, D. X., \& Cezar-Vaz, M. R. (2018). Sonolência diurna excessiva entre profissionais de enfermagem. Rev. enferm. UFPE on line, 1603-1609.

Souza, R. S., Araújo, F. L. Manzo, B. F., Marcatto, J. de O., Montenegro, L. C., Silva, P. R. M., \& Simão, D. A. da S. (2020). O cuidado na oncologia pediátrica: análise transversal da qualidade de vida de profissionais de enfermagem. Revista Brasileira de Enfermagem, 73.

Souza, V. S. de, Silva, D. S. da, Lima, L. V., Teston, E. F., Benedetti, G. M. dos S., Costa, M. A. R., \& Mendonça, R. R. (2018). Qualidade de vida dos profissionais de enfermagem atuantes em setores críticos. Revista cuidarte, 9(2), 2177-2186.

Trzimajewski, L. T., Pereira, A. D. A., Santini, R. G., \& Zamberlan, C. (2020). Avaliação da qualidade de vida dos profissionais de enfermagem em Unidade de Terapia Intensiva Adulto. Research, Society and Development, 9(10), e4289108168-e4289108168.

Viana, M. C. de O., Bezerra, C. M. B., Silva, K. K. M., Martino, M. M. F. de, Oliveira, A. P. C., Torres, G. V., Silva, D. M., \& Souza, A. M. L. (2019). Qualidade de vida e sono de enfermeiros nos turnos hospitalares. Revista Cubana de Enfermería, 35(2).

WHOQOL GROUP (1996). WHOQOL-BREF: Introduction, administration, scoring and generic version of the assessment. Geneva: World Health Organization. https://www.who.int/mental_health/media/en/76.pdf?ua=1. 